\title{
Respuesta biológica cuantitativa de dos depredadores (Heteroptera: Notonectidae) en el control larval de Aedes aegypti (Diptera: Culicidae)
}

\author{
Quantitative biological response of two predators (Heteroptera: Notonectidae) \\ in the larval control of Aedes aegypti (Diptera: Culicidae)
}

\author{
(iD) YURI AYALA-SULCA ${ }^{*}$, (iD PERCY COLOS-GALINDO ${ }^{1}$, (D) EDWIN \\ PORTAL-QUICAÑA ${ }^{1}$, (iD LUIS IBARRA-JUÁREZ², (D) REYNÁN CÓNDOR- \\ ALARCÓN ${ }^{1}$, (D) CARLOS CARRASCO-BADAJOZ ${ }^{1}$, iD RINA RAMÍREZ ${ }^{3}$
}

\begin{abstract}
${ }^{1}$ Universidad Nacional de San Cristóbal de Huamanga (UNSCH), Ayacucho, Perú.yuri.ayala@unsch.edu.pe* percy.colos@unsch.edu.pe,edwin.portal@ unsch.edu.pe, reynan.condor@unsch.edu.pe, carlos.carrasco@unsch.edu.pe ${ }^{2}$ Instituto de Ecologia: Xalapa, Veracruz, México,luis.ibarra@inecol.mx ${ }^{3}$ Universidad Nacional Mayor de San Marcos, Lima, Perú, rramirezm@unmsm.edu.pe
\end{abstract}

\begin{abstract}
Autor de correspondencia
Yuri Ayala Sulca Universidad Nacional de San Cristóbal de Huamanga (UNSCH), Ciudad Universitaria, Av. Independencia No. 401, Ayacucho, C.P. 05001, Perú, yuri.ayala@unsch.edu.pe*

\section{Citación sugerida}

AYALA-SULCA, Y.; COLOS-GALINDO, P.; PORTAL-QUICAÑA, E.; IBARRA-JUÁREZ, L.; CONDOR-ALARCÓN, R.; CARRASCOBADAJOZ, C.; RAMÍREZ, R. 2021. Respuesta biológica cuantitativa de dos depredadores (Heteroptera: Notonectidae) en el control larval de Aedes aegypti (Diptera: Culicidae). Revista Colombiana de Entomología 47 (2): e10535. https://doi.org/10.25100/socolen.v47i2.10535
\end{abstract}

Recibido: 19-Ago-2020

Aceptado: 14-Ene-2021

Publicado: 22-Jul-2021

Revista Colombiana de Entomología ISSN (Print): 0120-0488

ISSN (On Line): 2665-4385

https://revistacolombianaentomologia.univalle.edu.co

Open access

(c) (1)(2) B. BY-NC-SA 4.0

Publishers: Sociedad Colombiana de Entomología SOCOLEN (Bogotá, D. C., Colombia) https://www.socolen.org.co

Universidad del Valle (Cali, Colombia)

https://www.univalle.edu.co

(C) 2021 Sociedad Colombiana de Entomología SOCOLEN y Universidad del Valle - Univalle
Resumen: La depredación es un factor biótico que influye en la estructura y funcionamiento de los ecosistemas, su efecto se puede cuantificar a través de la respuesta funcional (RF) y numérica en un contexto de éxito-invasión. Utilizamos la RF para entender las relaciones coevolutivas y medir la respuesta biológica de los depredadores Notonecta peruviana y Buenoa fasciata en el control de larvas de cuarto estadio de Aedes aegypti $\left(\mathrm{F}_{1}\right)$ a diferentes densidades $(5,10,20,35,55$ y 80 larvas/litro). Los depredadores en ayuno (48 horas) se introdujeron a los envases de ensayo (un individuo por especie) y se evaluó a las 24 horas la RF, número de presas consumidas $(\mathrm{Ne})$, coeficiente de ataque $(a)$ y tiempo de manipuleo $(\mathrm{Th})$. $N$. peruviana y B. fasciata desarrollaron una RF tipo II en el consumo de larvas de Ae. aegypti $(p \leq 0,01)$ y ocuparon cercanos tiempos de manipuleo $(T h)(\mathrm{t}=-1,93 ; \mathrm{gl}=12 ; p=0,078)$. N. peruviana depredó $(\mathrm{Ne})$ el doble de larvas de Ae. aegypti $(p \leq 0,05)$ y mostró un mayor coeficiente de ataque $(a)$ en relación con $B$. fasciata $(\mathrm{t}=14,92 ; \mathrm{gl}=12 ; p=0,000)$. Los depredadores $N$. peruviana y $B$. fasciata desestabilizaron y consumieron en forma rápida las presas. $N$. peruviana devoró el doble de larvas atribuible al efecto depredador-presa de origen reciente en comparación al posible ajuste coevolutivo entre B. fasciata y Ae. aegypti. Sería importante considerar a las especies de depredadores en los programas de control larval de mosquitos aedinos.

Palabras clave: Depredación. Control Biológico. Notonecta peruviana. Buenoa fasciata. Aedes aegypti.

Abstract: Predation is a biotic factor that influences the structure and functioning of ecosystems, its effect can be quantified through functional (FR) and numerical response in a successful-invasion context. We use FR to understand the coevolutionary relationships, and measured the biological response of the predators Notonecta peruviana and Buenoa fasciata in the control of fourth-stage larvae of Aedes aegypti $\left(\mathrm{F}_{1}\right)$ at different densities $(5,10,20,35$, 55 and 80 larvae / liter). Fasting predators (48 hours) were introduced to the test containers (one individual per species) and the FR, the number of preys consumed $(\mathrm{Ne})$, the attack coefficient $(a)$ and handling time $(T h)$. $N$. peruviana and $B$. fasciata developed a type II FR in the consumption of larvae of Ae. aegypti $(p \leq 0.01)$, and occupied close handling times $(T h)$ $(\mathrm{t}=-1.93 ; \mathrm{df}=12 ; p=0.078) . N$. peruviana preyed $(\mathrm{Ne})$ twice as many larvae of Ae. aegypti $(p \leq 0.05)$ and showed a higher attack coefficient $(a)$ in relation to B. fasciata $(\mathrm{t}=14.92$; $\mathrm{df}=12 ; p=0.000)$. The predators $N$. peruviana and $B$. fasciata rapidly destabilized and consumed the preys. $N$. peruviana preyed twice as many larvae, attributable to the recent predator-prey effect compared to the possible coevolutionary adjustment between $B$. fasciata and $A e$ e aegypti. It would be important to consider the predator species in the vector control programs for aedine mosquitoes.

Keywords: Predation. Biologic control. Notonecta peruviana. Buenoa fasciata. Aedes aegypti.

\section{Introducción}

Los ecosistemas a nivel mundial no solo son afectados por el cambio climático y la acción antropogénica, sino por la proliferación de especies invasoras ecológica y ambientalmente perjudiciales que dañan y agotan rápidamente los recursos en una magnitud mayor que las especies nativas taxonómica y/o tróficamente similares (Dick et 
al. 2013; Cuthbert et al. 2019a). Especies altamente invasivas como el mosquito Aedes aegypti (Linnaeus, 1762) originario de África, que ingresó a América hace más de 500 años con la colonización europea, ha generado frecuentes e importantes epidemias de dengue y fiebre amarilla en varios países (Salvatella-Agrelo 1996; Simberloff y Gibbons 2004; Espinoza et al. 2005; Cabezas et al. 2015). En Perú fue reintroducido en 1984, estableciéndose en 269 distritos y 18 regiones (casi la tercera parte del país), con la consecuente expansión geográfica de enfermedades como el dengue, chikungunya, zika y fiebre amarilla (Cabezas et al. 2015; MINSA 2019). El 2015 fue reportado en la región selvática del Valle de los Ríos Apurímac, Ene y Mantaro (VRAEM), entre los departamentos de Ayacucho, Cusco y Junín (Brathwaite-Dick et al. 2012; Cabezas et al. 2015; Requena-Zuñiga et al. 2016).

Por más de 20 años en varios países del mundo, el control de larvas y adultos del mosquito se realizó con insecticidas como el temefos y malatión; a partir del 2005 en el Perú se encontró evidencia de resistencia a los piretroides permetrina y deltametrina (Cabezas et al. 2015). Se identificaron cinco haplotipos del mosquito relacionados con la variabilidad genética, la migración activa del vector y la migración pasiva mediada por la actividad humana (Leiva y Cáceres 2004; Yáñez et al. 2014) y se generó la necesidad de proponer nuevas alternativas de control. El empleo de insectos depredadores es una forma de interacción biótica clave que influye en la estructura y funcionamiento de los ecosistemas, y es relevante en el contexto del control biológico como una forma de resistencia biótica de las comunidades afectadas, determinantes en el éxito o fracaso de los invasores (Cuthbert et al. 2019a, 2019b). La densidad de larvas y pupas de los mosquitos en los ambientes acuáticos influyen en la cantidad, desarrollo, supervivencia y reproducción de los depredadores. Esta forma de interacción muestra su mejor ejemplo en la respuesta funcional (RF) y numérica (Smith y Smith 2009), conceptos ecológicos tradicionales aplicados por los ecólogos para cuantificar las fuerzas de interacción existente entre los consumidores (depredadores) y las presas en un contexto de éxito-invasión (Cuthbert et al. 2019a). Se han descrito tres tipos de RF, una lineal tipo I, otra hiperbólica de tipo II y la tipo III sigmoidea. Cada una describe un tipo de interacción entre el depredador y la presa. La RF tipo I se observa por lo general en animales filtradores; la del tipo II en depredadores invertebrados como los insectos (incluidos los parasitoides) y la del tipo III propia de depredadores vertebrados, y que se relaciona con el aprendizaje o por la posibilidad de cambiar una presa por otra (Fernández-Arhex y Corley 2004; Cuthbert et al. 2019a). En este sentido, la RF desarrollada por depredadores como Notonecta peruviana (Hungerford, 1933), insecto poco especialista y con amplia distribución geográfica en zonas tropicales y templadas del Perú y Sudamérica y Buenoa fasciata (Nieser, 1970), restringido a ambientes tropicales con dieta particular en larvas de mosquitos culícidos (Domínguez y Fernández 2009; Heckman 2011), ofrecen un método predictivo del posible impacto en organismos invasores como Ae. aegypti (presa), a ser considerado en los protocolos de evaluación y control vectorial de mosquitos de importancia médica. En la presente investigación, utilizamos la RF para entender las relaciones coevolutivas depredador-presa y la respuesta numérica para cuantificar el impacto biológico de los depredadores hemípteros $N$. peruviana y B. fasciata en el control de larvas de Ae. aegypti bajo condiciones controladas.

\section{Materiales y métodos}

Larvas de mosquito. Los huevos del mosquito Ae. aegypti procedieron del distrito de Kimbiri (La Convención - Cusco, Perú) (12 $37^{\circ} 07^{\prime \prime} \mathrm{S} ; 73^{\circ} 47^{\prime} 10^{\prime \prime} \mathrm{O}$; $\left.587 \mathrm{msnm}\right)$, recolectados en campo con ovitrampas de acuerdo con los métodos descritos por Fay y Eliason (1966) y Reiter et al. (1991) y trasladados en bolsas de papel al laboratorio de Zoología de la Facultad de Ciencias Biológicas de la Universidad Nacional de

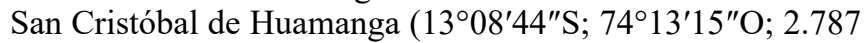
$\mathrm{msnm}$ ). En el laboratorio, las tiras de papel impregnadas con los huevos de Ae aegypti (especie única del género registrada en la zona) se sumergieron en bandejas de plástico (tamaño: $33 \times 22 \times 5 \mathrm{~cm})$ que contenían $850 \mathrm{~mL}$ de agua potable libre de cloro, atemperado a $22,5^{\circ} \mathrm{C}( \pm 0,5)$ y acondicionadas al interior de una cámara climatizada de crianza de insectos [temperatura $24,5{ }^{\circ} \mathrm{C}( \pm 0,5)$; humedad relativa $65 \%( \pm 2)$ y un fotoperiodo de 12:12 (Día: Noche)]. Las larvas emergidas fueron alimentadas con hojuelas trituradas de alimento para peces de acuario hasta alcanzar el estado de pupa. Obtenidos los estadios adultos, fueron acondicionados al interior de jaulas de crianza (tamaño: $61 \times 61 \times 61 \mathrm{~cm}$ ) proporcionándoles una solución azucarada al $10 \%$ en las primeras horas de emergencia (24 horas). Posteriormente, fueron alimentadas con sangre humana hasta lograr nuevas posturas y obtener la generación $\mathrm{F}_{1}$, utilizada en las pruebas experimentales (Ayala-Sulca et al. 2008). Las larvas emergidas de esta generación fueron criadas hasta alcanzar el cuarto estadio, necesario para las pruebas de depredación debido a la estabilidad fisiológica y morfológica que se presenta en él, además de mejores atributos de respuestas biológicas en ellas observables; pues los primeros estadios tienen corta duración y dificultan observar los resultados de modo confiable (Consoli y Oliveira 1994; Roberts 2014).

Se dispuso de un total de 1640 larvas de cuarto estadio distribuidas en densidades crecientes de 5, 10, 20, 35, 55 y 80 larvas/litro por depredador, en siete repeticiones con su respectivo control (totalizando 3280 larvas de Ae. aegypti para las dos especies en experimentación). Las larvas se introdujeron 24 horas antes de las pruebas en cada recipiente de evaluación para facilitar su adaptación a las nuevas condiciones del medio. Por precaución, las larvas estuvieron en observación para verificar su comportamiento, buen desarrollo fisiológico (movimientos ágiles típicos para la especie) y la posible mortandad (no registrada en los controles durante el experimento).

Depredadores heterópteros. Notonecta peruviana, se recolectó en charcas de agua estacional del centro poblado de Rancha, Chanchoccocha (Huamanga - Ayacucho, Perú) $\left(13^{\circ} 10^{\prime} 50^{\prime \prime} \mathrm{S} ; 74^{\circ} 15^{\prime} 51^{\prime \prime} \mathrm{O} ; 3.205 \mathrm{msnm}\right)$ y Buenoa fasciata, en estanques de cría de peces tropicales de la piscigranja de la municipalidad distrital de Pichari (La Convención - Cus-

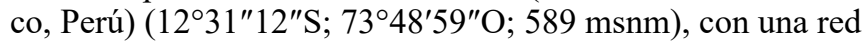
D-net y un "dipper" de $350 \mathrm{~mL}$ de capacidad. Se recolectaron 180 especímenes de $N$. peruviana y 350 especímenes de $B$. fasciata que fueron transportados al laboratorio en bolsas "whirl-pack" ${ }^{\circledR}$ acondicionadas en termos de plástico provistos de agua para evitar los cambios bruscos de temperatura y presión. En el laboratorio, los entomófagos fueron mantenidos por separado en peceras de vidrio de $25 \mathrm{~L}$ de capacidad conteniendo agua potable libre de cloro y alimentados " $a d$ libitum" con larvas de mosquitos culícidos hasta 48 horas 
antes de las pruebas de depredación. Los notonéctidos utilizados en el experimento fueron individualizados en vasos descartables abastecidos con $50 \mathrm{~mL}$ de agua para evitar el canibalismo.

En cada recipiente de prueba dispuesto con número creciente de larvas de cuarto estadio de Ae. aegypti (5, 10, 20, 35, 55, 80 larvas/litro), aspecto relevante del diseño experimental en la determinación del tipo de RF y necesario para estimar el número de presas consumidas por un depredador por unidad de tiempo, cuando se enfrenta a diferentes densidades de ofertas larvales (Fernández-Arhex y Corley 2004), se introdujo por separado un adulto del depredador $N$. peruviana y un adulto de $B$. fasciata sometidos a ayuno 48 horas antes, a fin de evidenciar su capacidad depredadora y maximizar sus respuestas. Transcurridas las 24 horas, se contabilizó el número de exuvias y/o larvas consumidas.

El experimento fue adecuado a un diseño experimental completamente aleatorizado (DCA), siendo cada densidad y especie de depredador evaluado en siete repeticiones con su respectivo control.

Cuantificación del impacto de los depredadores. La cuantificación del impacto biológico de los depredadores notonéctidos ( $N$. peruviana y $B$. fasciata) en el control de larvas de Ae. aegypti, fue estimada mediante la determinación del tipo de respuesta funcional. Posteriormente, se calculó el número de presas consumidas $(\mathrm{Ne})$, tasa de captura $(a)$ y tiempo de manipuleo $(T h)$.

Respuesta funcional. Con los resultados de mortalidad larval obtenidos en los ensayos de depredación, se realizó el análisis de regresión logística propuesta por Juliano (2001), a fin de establecer el tipo de respuesta funcional (RF) ajustada a uno de los modelos desarrollados por Holling (1959) y revisada por Pervez y Omkar (2005) [lineal en aumento (RF tipo I), curva cóncava (RF tipo II), curva sigmoidea (RF tipo III)]. Para lo cual se empleó el test de respuesta funcional de la librería Frair (Pritchard et al. 2018) y de R Core Team (2018).

Respuesta numérica. La respuesta numérica fue estimada por la ecuación aleatoria del disco de Holling para modelos cóncavos (RF tipo II) (Holling 1959; Juliano 2001) considerando el número de larvas depredadas o consumidas $(\mathrm{Ne})$ por especie de depredador y el resultado del análisis gráfico de la tendencia de curva de la respuesta funcional. En tanto que los parámetros de coeficiente de ataque o tasa de captura $(a)$, y tiempo promedio de manipuleo $(T h)$, fueron valoradas en función de la aplicación de la siguiente fórmula:

$$
N e=\frac{a N o T}{1+a N o t h}
$$

Dónde:

$\mathrm{Ne}$ : Número de larvas depredadas o consumidas

No: densidad de la presa ofertada inicialmente

$a$ : coeficiente de ataque constante o tasa de búsqueda instantánea

T: tiempo de contacto entre la presa y el depredador ( 24 horas o un día)

Th: Tiempo de manipulación de la presa.

Parámetros que fueron analizados y calculados por los aplicativos funcionales de los programas R Core Team (2018) y librería Frair (Pritchard et al. 2018).
Análisis de datos. Para cuantificar la respuesta biológica de los depredadores ( $N$. peruviana y B. fasciata) en el control de larvas de Ae. aegypti, se compararon la cantidad de presas consumidas $(\mathrm{Ne})$ por depredador en función del número de presas ofertadas $(\mathrm{No})$, aplicando la prueba no paramétrica de Mann-Whitney $(\alpha=0,05)$. Además, son reportadas las medias de larvas depredadas o consumidas $(\mathrm{Ne})$ por especie de depredador y su correspondiente desviación estándar $( \pm$ DE) para cada densidad de presa ofertada, los datos en este caso, fueron transformados a valores porcentuales. El coeficiente promedio de captura $(a)$ y tiempo promedio de manipuleo $(T h)$, fueron examinados estadísticamente mediante la prueba de $\mathrm{t}$ de Student $(\alpha=0,05)$ previa verificación de la distribución normal de los datos, con la finalidad de establecer las diferencias existentes entre los depredadores. Los análisis se realizaron en el programa estadístico R Core Team (2018).

\section{Resultados}

Respuesta funcional. En el análisis de la respuesta funcional, las curvas halladas (Fig. 1) demuestran que los depredadores $N$. peruviana y B. fasciata responden a una tendencia cóncava (RF de Tipo II según los modelos propuestos por Holling 1959) en el consumo de larvas de Ae. aegypti, que se caracteriza de modo general, porque las presas a altas densidades tienen menor probabilidad de ser atacadas que cuando están a bajas densidades. La figura 1 en análisis, denota claramente este comportamiento, a menores ofertas larvales (5 a 35 larvas) los entomófagos agotan las presas disponibles en el medio y muestran su máximo potencial de consumo o saturación ("plateau") a altas densidades (55 a 80 larvas), donde pese a existir exceso de presas solo devoran una proporción de ellas, equilibrando la depredación a una tasa de consumo que se hace constante. En este sentido, el análisis de regresión logística (Tabla 1) establece que el tipo de respuesta funcional que mejor se adecua al comportamiento de los notonéctidos evaluados en el consumo de larvas de Ae. aegypti, corresponde al Tipo II de tendencia cóncava $(p \leq 0,01)$ demostrado en el experimento.

Número de presas consumidas $(\mathrm{Ne})$. Congruente con los resultados de la RF, el entomófago $N$. peruviana consumió $(\mathrm{Ne})$ de 100 a $70 \%$ de las presas suministradas a las menores densidades (5 a 35 larvas) ( \pm DE: 5,00 $\pm 0,00$ a 24,57 $\pm 3,45$ larvas, respectivamente), en tanto que $B$. fasciata solo depredó de 59 a $44 \%$ del total ofertado ( \pm DE: $2,85 \pm 0,69$ a $15,57 \pm$ 1,81 larvas). A las densidades de 55 a 80 larvas de Ae. aegypti, $N$. peruviana no superó el $56 \%$ de depredación ( \pm DE: 30,71 $\pm 10,57$ a $35,85 \pm 9,85)$ y $B$. fasciata alcanzó un máximo de $28 \%$ de consumo larval ( \pm DE: $20,28 \pm 3,90$ a $22,42 \pm 3,20$ ) (Tabla 2), valores que denotan diferencias significativas entre las especies de entomófagos $(p \leq 0,05)$, siendo $N$. peruviana a la luz de estos resultados, la especie más promisoria en el consumo de larvas de Ae. aegypti en las seis densidades evaluadas.

Coeficiente de ataque (a) y tiempo de manipuleo (Th). En el coeficiente de ataque $(a)$, entendido como el intervalo que dispone el depredador en ubicar la presa e iniciar con el ataque en un ambiente controlado, $N$. peruviana $( \pm \mathrm{DE}: 1,081$ $\pm 0,030)$ responde de modo más sensible y eficiente que $B$. fasciata ( \pm DE: $0,580 \pm 0,084$ ) en la localización de las larvas de $A$ e. aegypti, respuesta altamente significativa según el 


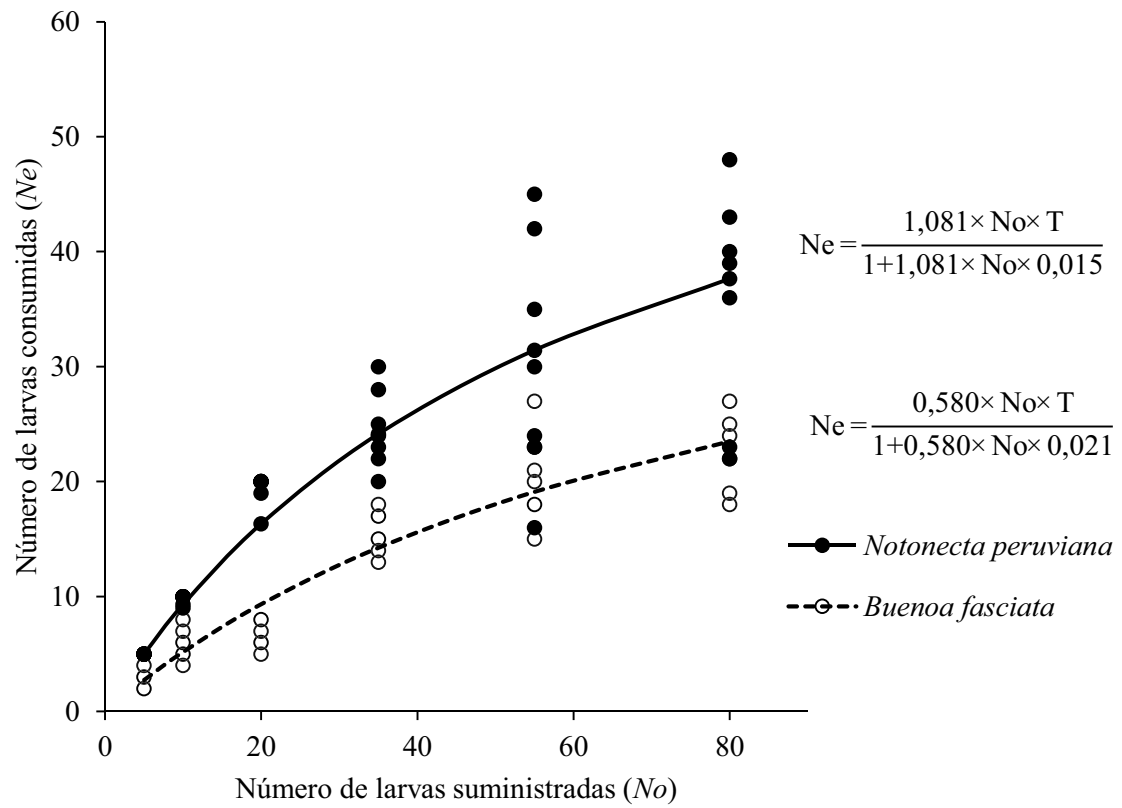

Figura 1. Respuesta funcional Tipo II desarrollada por Notonecta peruviana y Buenoa fasciata en el consumo de larvas de Aedes aegypti, según el análisis de regresión logística $(p \leq 0,01)$ adecuada al modelo cóncavo propuesto por Holling (1959) en la cuantificación del impacto depredador-presa.

Tabla 1. Análisis de regresión logística de la respuesta funcional tipo II desarrollada por Notonecta peruviana y Buenoa fasciata en el consumo de larvas de Aedes aegypti.

\begin{tabular}{lccccc}
\hline \multicolumn{1}{c}{ Depredador } & & Estimador & Error estándar & Valor de $\mathbf{z}$ & $\operatorname{Pr}(>|\mathbf{z}|)$ \\
\hline Notonecta peruviana & Densidad & $-0,037551$ & 0,002765 & $-13,581$ & $2,2 \times 10^{-16 * * *}$ \\
Buenoa fasciata & Densidad & $-0,0128959$ & 0,0022741 & $-5,6707$ & $1,422 \times 10^{-08 * * *}$ \\
\hline
\end{tabular}

*** Nivel de significancia $(p \leq 0,01$ altamente significativo)

Tabla 2. Número y porcentaje de larvas de Aedes aegypti consumidas en las pruebas de depredación de Notonecta peruviana y Buenoa fasciata, a seis densidades crecientes.

\begin{tabular}{|c|c|c|c|c|c|c|}
\hline \multirow{3}{*}{$\begin{array}{c}\text { Larvas } \\
\text { suministradas } \\
(\mathrm{No})\end{array}$} & \multicolumn{4}{|c|}{ Larvas consumidas $(\mathrm{Ne})($ media $\pm \mathrm{DE})$} & \multirow{3}{*}{$\boldsymbol{W}$} & \multirow{3}{*}{$p^{1}$} \\
\hline & \multicolumn{2}{|c|}{ Notonecta peruviana } & \multicolumn{2}{|c|}{ Buenoa fasciata } & & \\
\hline & $\mathrm{Ne}$ & $\%$ & $\mathrm{Ne}$ & $\%$ & & \\
\hline $5(7)$ & $5,00 \pm 0,00$ & $100 \pm 0$ & $2,85 \pm 0,69$ & $57 \pm 14$ &.-- &.-- \\
\hline $10(7)$ & $9,85 \pm 0,37$ & $99 \pm 4$ & $5,85 \pm 1,34$ & $59 \pm 13$ & 77,0 & 0,001 \\
\hline $20(7)$ & $19,85 \pm 0,37$ & $99 \pm 2$ & $6,85 \pm 1,21$ & $34 \pm 6$ & 77,0 & 0,001 \\
\hline $35(7)$ & $24,57 \pm 3,45$ & $70 \pm 10$ & $15,57 \pm 1,81$ & $44 \pm 5$ & 77,0 & 0,002 \\
\hline $55(7)$ & $30,71 \pm 10,57$ & $56 \pm 19$ & $20,28 \pm 3,90$ & $37 \pm 7$ & 68,5 & 0,047 \\
\hline $80(7)$ & $35,85 \pm 9,85$ & $45 \pm 12$ & $22,42 \pm 3,20$ & $28 \pm 4$ & 70,0 & 0,029 \\
\hline
\end{tabular}

Valores de $p$ obtenidos con la prueba de Mann-Whitney para dos depredadores diferentes y porcentaje de larvas consumidas en los tratamientos.

(No) Densidad de la presa suministrada inicialmente.

$(\mathrm{Ne})$ Número y porcentaje de larvas depredadas o consumidas.

análisis de la variable evaluada $(\mathrm{t}=14,92 ; \mathrm{gl}=12 ; p=0,000)$. En cuanto al tiempo de manipuleo $(T h)$, estimado como el tiempo que tarda el depredador en perseguir, dominar, consumir y digerir las presas, preparándose para la siguiente búsqueda y ataque, $N$. peruviana dispone de un tiempo en promedio de 0,015 (DE: $\pm 0,030)$ por día para alcanzar el máximo porcentaje de depredación de larvas de $A e$. aegypti, equivalente a 0,36 horas/día, tiempo que resulta ser próximo al que desarrolla $B$. fasciata ( \pm DE: $0,021 \pm 0,006$ ) cuya equivalencia a decir, es de 0,504 horas/día en el consumo de larvas de Ae. aegypti. No existen diferencias significativas para el tiempo de manipuleo $(\mathrm{t}=-1,93 ; \mathrm{gl}=12 ; p=0,078)$ que evidencien la superioridad de alguno de los dos depredadores en la manipulación de las larvas de Ae. aegypti, consecuentemente ambos depredadores ocupan tiempos similares en la depredación de las larvas (Tabla 3 ). 
Tabla 3. Coeficiente de ataque y tiempo de manipuleo de los depredadores Notonecta peruviana y Buenoa fasciata en el consumo de larvas de Aedes aegypti, estimado para un día de ensayo.

\begin{tabular}{cccccccc}
\hline \multirow{2}{*}{ Parámetros } & \multicolumn{2}{c}{ Especie de depredador (media $\pm \mathbf{D E})$} & & gl & $\boldsymbol{p}^{\mathbf{1}}$ \\
\cline { 2 - 3 } & Notonecta peruviana & Buenoa fasciata & & & & \\
\hline Coeficiente de ataque $(a)$ & $1,081 \pm 0,030$ & $0,580 \pm 0,084$ & & 14,92 & 12 & 0,000 \\
\hline Tiempo de manipuleo $(T h)$ & $0,015 \pm 0,005$ & $0,021 \pm 0,006$ & & $-1,93$ & 12 & 0,078 \\
\hline
\end{tabular}

${ }^{1}$ Nivel de significancia con la prueba de t-Student

\section{Discusión}

Nuestros resultados son consistentes en demostrar que los depredadores $N$. peruviana y $B$. fasciata desarrollan una respuesta funcional tipo II en el consumo de larvas de $A e$. aegypti al devorar un mayor número de presas a menores densidades y se evidencia el momento en el que alcanzan el límite superior de ataque o saturación (asintótica) en ajuste al modelo cóncavo propuesto por Holling (1959). Estos resultados corroboran los hallazgos de investigaciones previas para los notonéctidos en el control de larvas de mosquitos culícidos (Ellis y Borden 1970; Blaustein et al. 1995; Fischer et al. 2013; Buxton et al. 2020), tipo de respuesta funcional (modelo cóncavo) tipificado como denso dependiente inverso (o denso independiente) que pueden provocar la erradicación de las especies objeto de control (p.ej. larvas de Ae. aegypti) (Begon et al. 2000; Dick et al. 2013) y que según Pervez y Omkar (2005), se debe a las condiciones artificiales bajo las cuales fueron medidas. Sin embargo, las evidencias teóricas tipifican a las RF tipo II como inestables (o denso independientes), en la que los depredadores pueden llevar a la extinción de la presa, a bajas densidades, o dejarlas libre de control cuando se encuentran en densidades altas. En los sistemas acuáticos los controladores biológicos (como los insectos) ejercen un marcado impacto en la estructura biótica de las poblaciones de mosquitos de importancia médica (Cuthbert et al. 2019b); consecuentemente, la respuesta funcional demostrada proporciona información entomológica y ecológica importante que permite predecir el impacto de los entomófagos nativos en regiones biogeográficas tropicales y subtropicales del Perú y Sudamérica, en el control de larvas de especies invasoras como Ae. aegypti.

Debemos precisar que N. peruviana fue hallado en charcas temporales colonizadas por larvas de mosquitos del género Culex en valles interandinos y tropicales del departamento de Ayacucho (distribuidos entre los 800 a $3.200 \mathrm{msnm}$; Y. Ayala, observación personal), y se sometió a prueba frente a una presa no habitual (larvas de Ae. aegypti) lo que generó mayor impacto (depredación), aproximadamente el doble de lo que consume B. fasciata (especie tropical presente en la selva de Ayacucho, registrada entre los 589 a $1.000 \mathrm{msnm}$ colonizando regularmente el mismo hábitat de Ae. aegypti; Y. Ayala, observación personal). Esto se observa en la superior tendencia de curva de la RF, que equivale a un mayor impacto ecológico, eficiencia en la depredación y un comportamiento que sugiere una relación depredador-presa de origen reciente, caracterizado por generar una disminución rápida de la población objeto de control (Fernández-Arhex y Corley 2004; Dick et al. 2013). En tanto B. fasciata al ser un depredador que comparte el mismo hábitat de las larvas de Ae. aegypti, muestra menor efecto sobre la densidad larval relacionado probablemente con el equilibrio entomófago-presa, condicionado al consumo solo de lo necesario para satisfacer el requerimiento alimenticio evitando la extinción del recurso en control, que implica en términos biológicos eficiencia en la respuesta antidepredación de la presa y el posible ajuste coevolutivo entre ambos organismos establecido por selección natural a lo largo de cientos de miles de años, que evita la desaparición de una de las dos poblaciones (Fernández-Arhex y Corley 2004; Quiroz-Martínez et al. 2005).

En cuanto a la respuesta numérica, el análisis del coeficiente de ataque (a), demuestra que $N$. peruviana genera mayor efecto de embestida y mortalidad en las larvas de $A e$. aegypti en comparación a $B$. fasciata en todas las densidades evaluadas. Estos resultados sugieren a un potencial depredador en el control de larvas de mosquitos aedinos, en tiempos en que la lucha vectorial basada en los insecticidas es cuestionada. En cuanto al tiempo de manipuleo (Th), cada larva presa les demanda a ambos depredadores un tiempo relativamente similar, utilizado en perseguir, someter, consumir y prepararse para una nueva búsqueda de presas. De modo general y consistente con los resultados hallados en otras investigaciones (Dick et al. 2013; Cuthbert et al. 2019b) a medida que aumenta la densidad de las presas en el ambiente acuático, los depredadores las ubican y capturan fácilmente, pero la manipulación demanda el mismo tiempo. Por otro lado, cuando la abundancia de presas es infinita el depredador pasa su tiempo manipulándolas más no consumiéndolas (Pervez y Omkar 2005). En suma, $N$. peruviana demuestra mayor coeficiente de ataque $(a)$ y mayor número de presas consumidas (larvas de Ae. aegypti) (Ne) en relación a B. fasciata en el control de mosquitos aedinos, proporcionando soporte a la candidatura de este depredador como un potencial controlador biológico en los programas de control vectorial. Nuestros resultados son importantes porque permiten conocer el comportamiento y la potencialidad de los entomófagos como $N$. peruviana y $B$. fasciata en el control larval del mosquito Ae. aegypti, principal vector del dengue y la fiebre amarilla en la región biogeográfica del Valle de los Ríos Apurímac, Mantaro y Ene (en los departamentos de Ayacucho, Cusco y Junín en el Perú), insectos depredadores que se desarrollan en criaderos naturales y artificiales.

Los rasgos de alimentación generalistas de los notonéctidos son ventajosos, existen evidencias de que pueden depredar todos los estadios larvales de los mosquitos culícidos (su principal presa), colonizar por dispersión aérea de manera eficiente y natural los hábitats acuáticos donde se desarrollan los inmaduros y ser capaces de reproducirse en recipientes experimentales (Quiroz-Martínez et al. 2005; Cuthbert et al. 2019b), no acreditado en crianzas masivas con fines de 
liberación y posterior control en pruebas de campo según la literatura citada. Nuestros resultados indican que los notonéctidos evaluados pueden generar impacto importante en los estadios finales de desarrollo de los mosquitos. Sin embargo, se hace necesario probar alternativas biológicas mixtas como Bacillus thuringiensis var. israelensis en el control de las larvas de los mosquitos y mejorar el efecto regulador de las poblaciones en zonas propensas a enfermedades metaxénicas. Estos diseños reducen los gastos y repercuten en la economía, no requiriendo aplicaciones repetitivas de plaguicidas químicos, sobre todo cuando se logra el establecimiento de los depredadores y patógenos en los criaderos naturales, con beneficios ecológicos de bajo impacto en los ecosistemas y en lo social, por la disminución de la casuística de las enfermedades al controlar la densidad de los mosquitos adultos transmisores (Quiroz-Martínez et al. 2005; Fimia-Duarte et al. 2010).

La respuesta funcional y los parámetros que de ella derivan [número de presas consumidas $(\mathrm{Ne})$, coeficiente de ataque $(a)$ y tiempo de manipuleo $(T h)$ ], generan interés y controversia en términos de los resultados hallados y la interpretación que de ellos se deriva. Son modelos predictivos que ayudan a comprender los procesos de regulación implícitos en la relación plaga-controlador, jerarquiza los atributos y predice el grado de éxito de la medida de control y ayuda a desarrollar teorías más robustas que dan respaldo a la hora de la toma de decisiones para establecer nuevos programas basados en enemigos naturales (DeBach y Rosen 1991). Sin embargo, son necesarias pruebas complementarias que contribuyan a esclarecer la sincronización que debe existir entre las generaciones del insecto blanco y el controlador y las preferencias alimenticias del depredador, a fin de evitar el escape de la presa motivo de vigilancia o que pase a ser ignorada por el controlador en demerito de otra especie acompañante y sin importancia epidemiológica (Griffin 2014).

\section{Conclusiones}

El enfoque predictivo de la respuesta funcional tipo II desarrollada por los depredadores $N$. peruviana y B. fasciata demostró ser potencialmente desestabilizador en el consumo de larvas de Ae. aegypti en condiciones de laboratorio. N. peruviana genera un mayor impacto ecológico al consumir el doble de la densidad de larvas del mosquito aedino, atribuible al efecto depredador-presa de origen reciente en comparación al posible ajuste coevolutivo desarrollado entre $B$. fasciata y Ae. aegypti. Ambos depredadores reportan alto consumo de presas $(\mathrm{Ne})$, elevado coeficiente de ataque $(a)$ y tiempos de manipuleo $(T h)$ relativamente bajos que las catalogan como especies promisorias e importantes componentes a considerar en los programas de control vectorial de larvas de mosquitos aedinos.

\section{Agradecimiento}

Nuestro agradecimiento a la Unidad de Posgrado de la Facultad de Ciencias Biológicas de la Universidad Nacional Mayor de San Marcos (Lima-Perú), por el apoyo científico en la conceptualización y desarrollo de la investigación.

\section{Literatura citada}

AYALA-SULCA, Y. O.; IBARRA-JUAREZ, L.; GRIECO, J. P.; ACHEE, N.; MERCADO-HERNANDEZ, R.; FERNÁNDEZ-SALAS, I. 2008. Respuesta conductual de Aedes aegypti (Linnaeus, 1762) frente a adulticidas piretroides de uso frecuente en salud pública. Revista Peruana de Medicina Experimental y Salud Pública 25 (1): 26-34. http://www.scielo.org.pe/scielo. php?script=sci_arttext\&pid=S1726-46342008000100005

BEGON, M.; HARPER, J.; TOWNSEND, C. 2000. Ecología: Individuos, poblaciones y comunidades. Ed. Omega. Barcelona-España. $450 \mathrm{p}$.

BLAUSTEIN, L.; KOTLER, B. P.; WARD, D. 1995. Direct and indirect effects of a predatory backswimmer (Notonecta maculata) on community structure of desert temporary pools. Ecological Entomology 20 (4): 311-318. https://doi.org/10.1111/j.1365-2311.1995.tb00462.x

BRATHWAITE-DICK, O.; SAN MARTÍN, J. L.; MONTOYA, R. H.; DEL DIEGO, J.; ZAMBRANO, B.; DAYAN, G. H. 2012. The history of dengue outbreaks in the Americas. The American Journal of Tropical Medicine and Hygiene 87 (4): 584-593. https://doi.org/10.4269/ajtmh.2012.11-0770

BUXTON, M.; CUTHBERT, R. N.; DALU, T.; NYAMUKONDIWA, C.; WASSERMAN, R. J. 2020. Predator density modifies mosquito regulation in increasingly complex environments. Pest Management Science 76 (6): 2079-2086. https://doi.org/10.1002/ ps.5746

CABEZAS, C.; FIESTAS, V.; GARCÍA-MENDOZA, M.; PALOMINO, M.; MAMANI, E.; DONAIRES, F. 2015. Dengue en el Perú: A un cuarto de siglo de su reemergencia. Revista Peruana de Medicina Experimental y Salud Pública 32 (1): 146-156. https://doi.org/10.17843/rpmesp.2015.321.1587

CONSOLI, R. A. G. B.; OLIVEIRA, R. L. de. 1994. Principais mosquitos de importância sanitária no Brasil. Editora FIOCRUZ. Rio de Janeiro. 225 p. https://www.arca.fiocruz.br/handle/icict/2708

CUTHBERT, R. N.; CALlAGHAN, A.; DICK, J. T. A. 2019a. A novel metric reveals biotic resistance potential and informs predictions of invasion success. Scientific Reports 9 (1): 1-11. https://doi.org/10.1038/s41598-019-51705-9

CUTHBERT, R. N.; DALU, T.; WASSERMAN, R. J.; CALLAGHAN, A.; WEYL, O. L. F.; DICK, J. T. A. 2019b. Using functional responses to quantify notonectid predatory impacts across increasingly complex environments. Acta Oecologica 95: 116-119. https://doi.org/10.1016/j.actao.2018.11.004

DeBACH, P.; ROSEN, D. 1991. Biological control by natural enemies. 2 edition. Cambridge University Press. Cambridge-Inglaterra. $456 \mathrm{p}$.

DICK, J.; GALLAGHER, K.; AVLIJAS, S.; CLARKE, H.; LEWIS, S.; LEUNG, S.; MINCHIN, D.; CAFFREY, J.; ALEXANDER, M.; MAGUIRE, C.; HARROD, C.; REID, N.; HADDAWAY, N.; FARNSWORTH, K.; PENK, M.; RICCIARDI, A. 2013. Ecological impacts of an invasive predator explained and predicted by comparative functional responses. Biological Invasions 15: 837-846. https://doi.org/10.1007/s10530-0120332-8

DOMÍNGUEZ, E.; FERNÁNDEZ, H. R. 2009. Macroinvertebrados bentónicos sudamericanos: Sistemática y biología. Fundación Miguel Lillo. Tucumán-Argentina. 654 p. http:// www.lillo.org.ar/publicaciones/especiales/macroinvertebradosbentonicos-sudamericanos

ELLIS, R. A.; BORDEN, J. H. 1970. Predation by Notonecta undulata (Heteroptera: Notonectidae) on Larvae of the Yellow-Fever Mosquito. Annals of the Entomological Society of America 63 (4): 963-973. https://doi.org/10.1093/aesa/63.4.963

ESPINOZA, M.; CABEZAS, C.; RUIZ, J. 2005. Un acercamiento al conocimiento de la fiebre amarilla en el Perú. Revista Peruana de Medicina Experimental y Salud Pública 22 (4): 308-315. http:// www.scielo.org.pe/scielo.php?script $=$ sci_arttext\&pid=S172646342005000400009\&lng=es\&tlng=es 
FAY, R.; ELIASON, D. 1966. A preferred oviposition site as a surveillance method for Aedes aegypti. Mosquito News 26 (4): 531535. https://www.biodiversitylibrary.org/content/part/JAMCA/ MN_V26_N4_P531-535.pdf

FERNÁNDEZ̄-ARRHEX, V.; CORLEY, J. C. 2004. La respuesta funcional: Una revisión y guía experimental. Ecología Austral 14 (1): 83-93. https://www.fcnym.unlp.edu.ar/catedras/ecopoblaciones/ TP/Respuesta\%20funcional.pdf

FIMIA-DUARTE, R.; DÍAZ, Z. M.; RAMOS, R. Q.; REID, J. W.; SANTANDER, C. E.; VICTORES, L. S. 2010. En torno a la depredación experimental de larvas de mosquitos por el copépodo Mesocyclops aspericornis (Copepoda: Cyclopoida). Revista Electrónica de Veterinaria 11 (38): 1- 7. https://www.redalyc. org/pdf/636/63613140022.pdf

FISCHER, S.; ZANOTTI, G.; CASTRO, A.; QUIROGA, L.; VARGAS, D. 2013. Effect of habitat complexity on the predation of Buenoa fuscipennis (Heteroptera: Notonectidae) on mosquito immature stages and alternative prey. Journal of Vector Ecology 38: 215-223. https://doi.org/10.1111/j.1948-7134.2013.12033.x

GRIFFIN, L. 2014. Laboratory evaluation of predation on mosquito larvae by Australian mangrove fish. Journal of Vector Ecology 39 (1): 197-203. https://doi.org/10.1111/j.1948-7134.2014.12087.x

HECKMAN, C. W. 2011. Encyclopedia of South American Aquatic Insects: Hemiptera - Heteroptera: Illustrated keys to known families, genera, and species in South America. Springer Países Bajos. $679 \mathrm{p}$.

HOLLING, C. S. 1959. Some characteristics of simple types of predation and parasitism. The Canadian Entomologist 91 (7): 385398. https://doi.org/10.4039/Ent91385-7

JULIANO, S. A. 2001. Nonlinear curve fitting: Predation and functional response curves. Scheiner, S. M.; Gurevitch, J. (Eds.). Design and analysis of ecological experiments. 2nd. ed. Oxford University Press. Oxford pp. 178-196.

LEIVA, N.; CÁCERES, O. 2004. Variabilidad genética de $A e$ des aegypti en algunas áreas del Perú usando single stranded conformational polymorphism (SSCP). Revista Peruana de Medicina Experimental y Salud Pública 21 (3): 157-66. http://www.scielo.org.pe/scielo.php?script=sci_arttext\&pid= S1726-46342004000300007\&lng=es\&nrm=iso

MINSA, MINISTERIO DE SALUD. 2019. Boletín Epidemiológico del Perú: Semana Epidemiológica 07-2019 [Internet]. Lima, Perú: Centro Nacional de Epidemiología, Prevención y Control de Enfermedades. https://www.dge.gob.pe/portal/docs/vigilancia/boletines/2019/07.pdf

PERVEZ, A.; OMKAR. 2005. Functional responses of coccinellid predators: An illustration of a logistic approach. Journal of Insect Science 5: 1-6. https://doi.org/10.1093/jis/5.1.5

PRITCHARD, D. W.; PATERSON, R. A.; BOVY, H. C.; BARRIOS-O'NEILL, D. 2018. Frair: An R package for fitting and comparing consumer functional responses. Methods in Ecology and Evolution 8 (11): 1528-1534. https://doi.org/ 10.1111/2041-210X.12784

QUIROZ-MARTÍNEZ, H.; RODRÍGUEZ-CASTRO, V. A.; SOLÍS-ROJAS, C.; MALDONADO-BLANCO, M. G. 2005. Predatory capacity and prey selectivity of nymphs of the dragonfly Pantala hymenaea. Journal of the American Mosquito Control Association 21 (3): 328-330. https://doi.org/ 10.2987/8756-971X(2005)21[328:PCAPSO]2.0.CO;2

R CORE TEAM. 2018. A language and environment for statistical computing. [Internet]. Vienna, Austria: R Foundation for Statistical Computing. https://www.r-project.org/
REITER, P.; AMADOR, M. A.; COLON, N. 1991. Enhancement of the CDC ovitrap with hay infusions for daily monitoring of Aedes aegypti populations. Journal of the American Mosquito Control Association 7 (1): 52-55. https://pubmed.ncbi.nlm.nih. gov/2045808/

REQUENA-ZUÑIGA, E.; MENDOZA-URIBE, L.; GUEVARA-SARAVIA, M. 2016. Nuevas áreas de distribución de Aedes aegypti en Perú. Revista Peruana de Medicina Experimental y Salud Pública 33 (1): 171-172. https://doi.org/10.17843/rpmesp. 2016.331.1804

ROBERTS, D. 2014. Mosquito larvae change their feeding behavior in response to kairomones from some predators. Journal of Medical Entomology 51 (2): 368-374. https://doi.org/10.1603/ ME13129

SALVATELLA-AGRELO, R. 1996. Aedes aegypti, Aedes albopictus (Diptera, Culicidae) y su papel como vectores en las Américas. La situación de Uruguay. Revista Médica del Uruguay 12: 28-36. http://www.rmu.org.uy/revista/1996v1/art5.pdf

SIMBERLOFF, D.; GIBBONS, L. 2004. Now you See them, now you don't! - Population Crashes of Established Introduced Species. Biological Invasions 6 (2): 161-172. https://doi.org/ 10.1023/B:BINV.0000022133.49752.46

SMITH, R. L.; SMITH, T. M. 2009. Ecología. Sexta edición. Pearson Education. España. 775 p. https://bgf-info9.webnode.com/ files/200000679-4ac514ac53/Ecologia.6ed.Smith.PDF.pdf

YÁÑEZ, P.; MAMANI, E.; VALLE, J.; GARCÍA, M. P.; LEÓN, W.; VILLASECA, P.; TORRES, D.; CABEZAS, C. 2014. Variabilidad genética del Aedes aegypti determinada mediante el análisis del gen mitocondrial ND4 en once áreas endémicas para dengue en el Perú. Revista Peruana de Medicina Experimental y Salud Pública 30 (2): 246-250. https://doi.org/10.17843/ rpmesp.2013.302.199

\section{Origen y financiación}

El estudio fue financiado por la Oficina General de Investigación e Innovación y el Vicerrectorado de Investigación de la Universidad Nacional de San Cristóbal de Huamanga a través del Fondo de Ayuda Financiera a la Investigación (Ex FEDU).

\section{Contribuciones de los autores}

$Y A S, P C G, C C B$ y RRM han participado en la concepción y diseño del artículo; YAS, PCG, LIJ y EPQ colaboraron en la definición del contenido intelectual y la búsqueda de literatura; YAS, PCG, RCA y CCB realizaron los estudios experimentales y la obtención de datos; YAS, RCA y CCB participaron en el análisis de los datos experimentales; $R C A$ y $C C B$ realizaron el análisis estadístico; YAS, RCA y CCB fueron responsables de preparar el manuscrito; YAS, PCG, EPQ, LIJ y $R R M$ realizaron la edición del manuscritos. En la revisión y redacción final del manuscrito participaron todos los miembros del equipo de investigación.

\section{Conflictos de interés}

Los autores declaramos no tener conflictos de intereses. 